\title{
Development of a mobile HTS cryogenic oscillator
}

\author{
O. d'Allivy Kelly ${ }^{1}$, Y. Lemaître ${ }^{1}$, B. Marcilhac ${ }^{1}$ \\ ${ }^{1}$ Unité Mixte de Physique, CNRS, Thales, Univ. Paris- \\ Sud, Université Paris-Saclay, 91767, Palaiseau, France \\ olivier.dallivykelly@thalesgroup.com
}

\author{
D. Chaudy ${ }^{2,3}$, J.-M. Hode ${ }^{2}$ \\ ${ }^{2}$ Thales Systèmes Aéroportés \\ 75-77 Avenue Marcel Dassault, 33700, Mérignac, France
}

\author{
O. Llopis ${ }^{3}$ \\ ${ }^{3}$ LAAS-CNRS, Université de Toulouse, CNRS \\ 7 avenue du Colonel Roche, 31031, Toulouse, France
}

\begin{abstract}
High-temperature superconductors (HTS) have shown their potential for analog signal processing at microwave frequencies; they provide high $Q$ resonators, the most critical component of an efficient low phase noise oscillator. Thus, a strategy to outperform state of art quartz oscillators could consist in integrating, in a monolithic block, a planar HTS resonator and a cryo-compatible transistor to build a $1 \mathrm{GHz}$ oscillator.

However, mobile systems may require reducing hardware size, weight and power consumption while meeting high performances, controlled costs and reliability needs. We discuss these questions and present our approach to validate the integration of HTS based oscillators into embedded systems.
\end{abstract}

Keywords-Cryogenics; HTS; Oscillators

\section{INTRODUCTION}

There is a need for high frequency oscillators exceeding quartz performance while meeting mobility constraints of embedded applications (land, naval, airborne systems...). In this respect, HTS technology has many advantages (very high Q factor resonators, reproducibility, frequency accuracy...) that lead now to design high performance HTS oscillators [1]. Even with the very high Q factor of a HTS resonator, the expected phase noise has never been observed on operational oscillators. 3D resonators are vibration sensitive and 2D resonators have been usually tested at too high frequencies.

Our goal is to outperform the specifications of acoustic resonators. To do so, we chose to operate planar resonators at $1 \mathrm{GHz}$, which has the advantages of a Q higher than 100000 , lower multiplication rank and availability of low $1 / \mathrm{f}$ noise transistors. Moreover, in order to fully benefit from the low noise floor level at $70 \mathrm{~K}$, we also cool the entire feedback loop including a low-temperature RF amplifier [2].

\section{THE TECHNOLOGY PUSH LIMITS}

Since the discovery of $\mathrm{YBa}_{2} \mathrm{Cu}_{3} \mathrm{O}_{7-\delta}$ (YBCO), more than 30 years ago, the HTS circuits have proven their relevance for high performance RF signal processing. Different circuits (filter banks, multiplexers, ...) have already been designed and characterized, presenting very high performances [3.4].

However, there are still many challenges to overcome for this technology to become a product. Indeed, it is not possible to only focus on the HTS circuit without considering the operational requirements, for example a compact cryogenic system is mandatory for embedded applications. Thus, to be developed, an HTS oscillator must be considered as a whole and not only in terms of performance.

\section{A GLOBAL APPROACH}

Specific applications (military and aerospace), require reducing hardware Size, Weight and Power consumption (SWaP) while meeting high performances, controlled costs, lifetime and reliability needs. Furthermore, due to large R\&D costs, HTS devices development requires to address different domains such as RF filters, multiplexers, oscillators but also detectors (bolometers, superconducting quantum interference devices, microwave kinetic inductance detectors, etc.).

\section{Cryogenic cooling}

HTS devices working temperature (between $60 \mathrm{~K}$ and $80 \mathrm{~K}$ ) is reached and sustained by a cryogenic cooler. It seems difficult to develop from scratch specific cryogenic coolers for each use case. A better idea would consist in re-using coolers employed with infrared sensors as these devices have already shown their performances in terms of reliability and compactness.

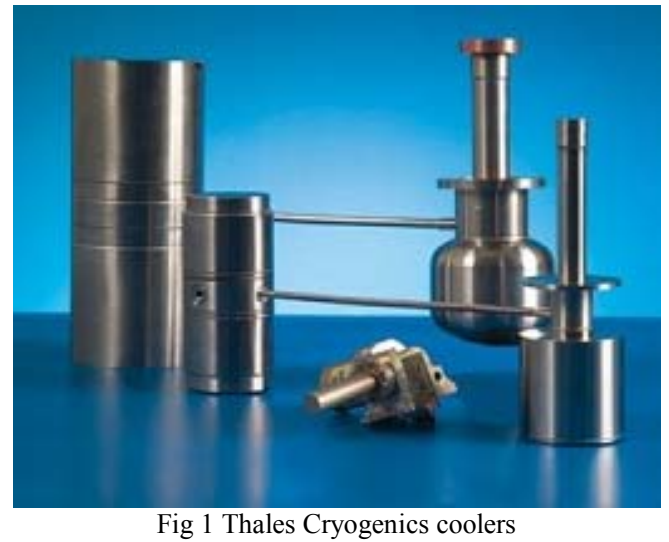

source: www thales-cryogenics com

We currently work with cooler devices provided by Thales Cryogenics, one of the European leaders in cryogenic 
technology. The best tradeoff between operational constraints and specific needs must be found among the different kinds of coolers: rotary monoblocs, linear split Stirling and pulse tubes [5].

In the case of the oscillator, the vibration noise insulation is critical, therefore we have excluded rotary cooler despite its compactness because the cold finger is directly connected to the motor.

The main advantage of a pulse tube on Stirling machines is that there are no moving parts in the cold finger, so less vibration. On the contrary, Stirling benefit of a smaller size and a better efficiency. An active vibration reduction system can be implemented to these two coolers.

\section{Cryopackaging}

In order to make mobile devices, standalone dewar (the vacuum chamber that contains HTS circuits) must be developed.

This process has to take into acount many constraints (static vacuum, thermal losses, $\mathrm{AC} / \mathrm{DC}$ feedthrough, lifetime,...). A standalone compact cryogenic system for RF filter has already been developed by Thales (Fig 2). We are now working to standardize the design and make it compatible with HTS oscillators.

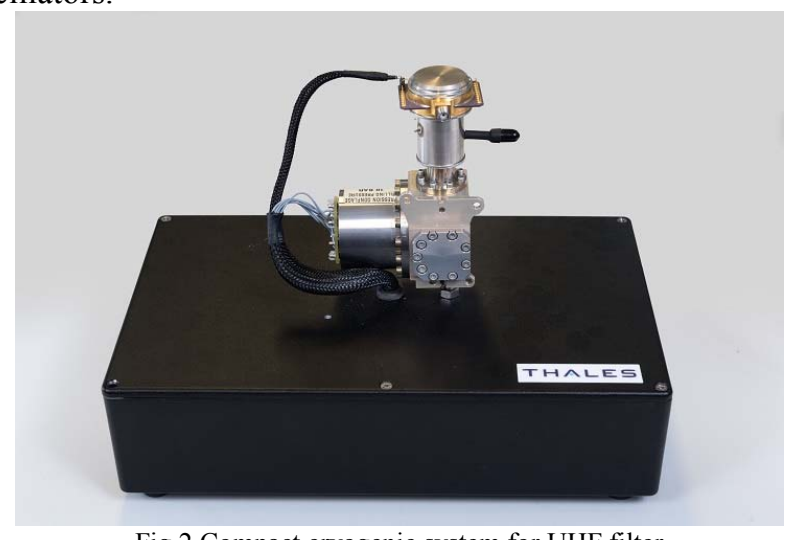

Fig 2 Compact cryogenic system for UHF filter

\section{HTS planar resonator circuit}

Another key point is the reproducibility of HTS planar resonator performances ( $Q$ factor, center frequency).

In this respect, it is necessary to have access to a HTS foundry providing industrial grade YBCO films and stabilized technological processes (lithography, etching, cutting...).

The resonator we use (Fig 3) is designed as a folded half wavelength line. Instead of the classical hairpin shape, we optimized the geometry in a nearly closed $\mathrm{C}$ in order to: minimize the size (large pads in the capacitive part of the line), improve the power handling capability (large half circle for the inductive part of the line where the current flows), and minimize the radiated energy (very short electric dipole corresponding to the gap).

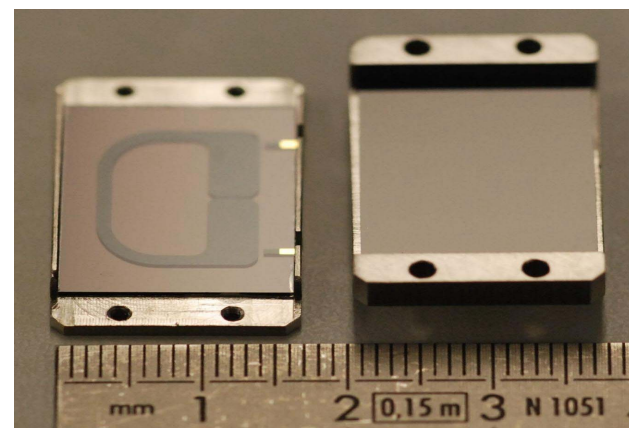

Fig 3 Superconducting resonator: YBCO film (700 $\mathrm{nm}$ thick) deposited on a magnesium oxide substrate $(500 \mu \mathrm{m}$ thick $)$

\section{CONCLUSION}

The development of a mobile HTS oscillator is part of a broader framework for the development of HTS technology. It is necessary to adopt a global approach if we want to address use cases. Our goal is to succeed in crossing the innovation gap with a full set of validated industrial processes to build the embedded systems fitting the military and aerospace markets' requirements in terms of quality and quantity.

\section{ACKNOWLEDGMENT}

This work is supported by the Direction Générale de l'Armement (DGA).

\section{REFERENCES}

[1] Mage J.C, Marcilhac B, Poulain M, Lemaitre Y., Kermorvant J, Lesage J.M., "Low noise oscillator based on 2D superconducting resonator", presented at the IFCS2011, San Fransisco, CA, USA, 2011

[2] D. Chaudy, O. Llopis, B. Marcilhac, Y. Lemaitre, O. d'Allivy Kelly, J.M. Hode "Active devices choice and design of an all cryogenic superconductor resonator oscillator" submitted to IFCS2017, Besançon, France, 2017

[3] M. J. Lancaster, "Passive Microwave Device Applications of HighTemperature Superconductors", Cambridge University Press, 2006

[4] J-S. Hong, "Recent development in superconducting filters, International Workshop on Microwave Filters", presented at International Workshop on Microwave Filters, France, 2006

[5] D. Willems, J. Mullié, T. Benschop, G. de Jonge "High-reliability and high-efficiency cryocoolers for HTS Applications" presented at the International Workshop on Cooling System for HTS Applications (IWC-HTS), Matsue, Japan, 2015 